\title{
Perceived body size and desire for thinness of young Japanese women: a population-based survey
}

\author{
F. Hayashi ${ }^{1,2} *$ H. Takimoto ${ }^{3}$, K. Yoshita ${ }^{1}$ and N. Yoshiike ${ }^{1}$ \\ ${ }^{1}$ Centre for Collaborative Research, National Institute of Health and Nutrition, 1-23-1 Toyama Shinjyuku-ku, \\ Tokyo, 1628636 Japan \\ ${ }^{2}$ Graduate School of Medical and Dental Science, Tokyo Medical and Dental University, 1-5-45 Yushima, \\ Bunkyo-ku, Tokyo, 1138519 Japan \\ ${ }^{3}$ Department of Health Promotion and Research, National Institute of Public Health, 2-3-6 Minami, \\ Wako-shi, Saitama, 3510197 Japan
}

(Received 22 December 2005 - Revised 30 June 2006 - Accepted 7 July 2006)

\begin{abstract}
The present study describes findings in relation to perceived body size and 'desire for thinness' by age and residential areas ('metropolitan areas', 'large cities', 'small cities' and 'towns') among young Japanese women. Data on 1731 non-pregnant, non-lactating women aged 15-39 years from the 1998 National Nutrition Survey of Japan were used. Current body size was evaluated by BMI percentiles (lean, $<5$ th; underweight, 5th $\leq$ BMI $<25$ th; normal, 25th $\leq$ BMI $<75$ th; overweight, 75th $\leq$ BMI $<95$ th; obese, $\geq 95$ th), calculated for 5-year age groups. Perceived body size was obtained by self-report. We defined 'overestimation' as non-overweight, non-obese women who perceived themselves as being 'overweight' or 'obese'. Desired body size was evaluated by applying the desired BMI to these cut-off points. Of all the women, $48.4 \%$ perceived themselves as being 'overweight' or 'obese', and $43.7 \%$ desired a 'lean' or 'underweight' body size. Adjusted for the current BMI, the OR for 'overestimation' calculated by a logistic regression model was significantly elevated in the 15-19-year age group (OR 2.79; 95\% CI 1.76, 4.43), compared with the 25-29-year age group. The OR for 'desire for thinness' was significantly high in the 35-39-year age group (OR 2.74; $95 \%$ CI $1.93,3.89$ ) and the 15-19-year age group (OR 2.26; $95 \%$ CI 1.57, 3.24). Women living in metropolitan areas had higher OR for 'desire for thinness' (but not for 'overestimation') than did women in towns (OR 1.47; $95 \%$ CI 1.05, 2.07). The findings suggest the nature of excessive weight concerns of young women in Japan; thus efforts to control such health-risk behaviours at a national level are urgent.
\end{abstract}

Underweight: Weight perception: Young women: Japan: Geographical differences

Almost all industrialised countries face epidemics of obesity, and Japan is no exception. A recent report from the WHO documented that the number of overweight adults worldwide has exceeded one billion, and that at least 300 million of these were clinically obese (World Health Organization, 2003). In Japan, the prevalence of overweight (BMI $\geq 25 \mathrm{~kg} / \mathrm{m}^{2}$ ) in adult men has risen approximately $10 \%$ in the last two decades according to the National Nutrition Survey of Japan (NNS-J) (Yoshiike et al. 2002). One in every five men aged 20-29 years, and one in every three or four men aged over 30 years are now overweight (Ministry of Health, Labour and Welfare, 2004). However, the trend in Japanese women differs from men, because the prevalence of underweight is increasing, especially in young women (Ministry of Health, Labour and Welfare, 2004).

Overweight or obesity are known to be associated with an increased risk of chronic diseases, such as type 2 diabetes (Manson et al. 1990; Chan et al. 1994), CVD (Manson et al. 1990; Wilson et al. 2002), and certain forms of cancer (Calle \& Thun, 2004), due to metabolic changes caused by excess fat. However, underweight is also associated with negative health consequences, such as nutritional deficiency (Russell et al. 1994), osteoporosis (Blum et al. 2001), and unfavourable pregnancy outcomes in women of childbearing age (Edwards et al. 1979). As in obesity, underweight is also an important risk factor to increased mortality risk in Japanese (Tsugane et al. 2002).

In Japan, we have a 10 -year national plan to promote the health of the Japanese population named 'Health Japan 21', which was released in 2000. One of the plan's objective aims to decrease the prevalence of underweight (BMI $<18.5 \mathrm{~kg} / \mathrm{m}^{2}$ ) in women aged 20-29 years to less than $15 \%$, and to increase the proportion of individuals who are aware of their own optimal weight to $90 \%$ or more by 2010 (Ministry of Health, Labour and Welfare, 2000a). However, the recent data from the NNS-J showed little change in the prevalence of underweight from the baseline (Ministry of Health, Labour and Welfare, 2004).

Most previous studies regarding desire for thinness in young women are from Western societies, where most women are at risk of overweight or obesity rather than underweight. Another limitation is that previous studies on body dissatisfaction or other related issues in Japanese women were from small

\footnotetext{
Abbreviations: NNS-J, National Nutrition Survey of Japan.

*Corresponding author: Ms Fumi Hayashi, fax +31 33202 3278, email fumi-nns@nih.go.jp
} 
convenient samples or specific age groups such as adolescents or college students (Watanabe et al. 2003; Wardle et al. 2005). Even in the USA where many pioneering work in this area has been documented, a recent population-based study on the prevalence of overweight misperceptions and weight-control behaviours among normal-weight subjects included only adolescents, and used the self-reported height and weight measurements for the analyses (Talamayan et al. 2006). Other population-based studies were looking at misperceptions of body size as barriers to reducing overweight rather than underweight (Paeratakul et al. 2002; Kuchler \& Variyam, 2003).

Takimoto et al. (2004) reported that the decrease in BMI from 1976 to 2000 was prominent among young Japanese women they studied (15-19, 20-24 and 25-29 years of age). However, BMI changes and the prevalence of underweight of women aged 15-19 years living in metropolitan areas predominated those living in smaller towns. That is, there was a difference in the prevalence of underweight by geographical region. McLaren \& Gauvin (2003) reported that living among thinner women increases the likelihood of reporting dissatisfaction with body image. Therefore, we assumed that women living in metropolitan areas and large cities were more likely to misperceive their body size and report stronger desire for thinness compared with women living in smaller towns.

In accordance with the result of the previous studies and assumptions we made, the objective of the present study was to describe the situation of perceived body size and desire for thinness among young Japanese women, by using datasets from a population-based survey. Furthermore, we evaluated relationships of perceived body size and desire for thinness with age and residential areas.

\section{Methods}

\section{Survey sample and methodology}

Datasets of the NNS-J in 1998 were used in the present study (Ministry of Health, Labour and Welfare, 2000b). The NNS-J was initiated in 1945 to assess the nutritional status and socioeconomic status of the Japanese population to obtain urgently needed food supplies from other countries, but it now monitors and assesses the dietary intakes and health outcomes of the population. Each November, approximately 15000 members from nearly 5000 households in randomly sampled census units are surveyed by the local public health centres under the supervision of the Japanese Ministry of Health, Labour, and Welfare. There are three major components in the NNSJ. One is the physical examination survey, in which all participants aged 1 year and older are invited to have their height and weight measured at local public health centres in the selected survey area. Blood pressure measurements and a $1 \mathrm{~d}$ step tests using a pedometer are recorded for participants aged 15 years and older. Blood tests of haematological parameters, lipid profiles, and glucose levels are only conducted for adults aged 20 years and older, and blood is drawn $3 \mathrm{~h}$ or more after the last meal. All physical measurements are conducted by trained public health nurses, according to the measurement protocol in the NNS-J (Ministry of Health, Labour and Welfare, $2000 \mathrm{~b}$ ). The second component is the $1 \mathrm{~d}$ weighed dietary record of all members of the survey household. The third component is the dietary questionnaire, which all participants aged
15 years and older are asked to fill in. The contents of the dietary questionnaires differ each year. Before the analyses, we received permission of the use of NNS-J datasets from the Ministry of Health, Labour and Welfare.

We evaluated 1731 non-pregnant and non-lactating women aged 15-39 years for whom information on height and weight measurements and dietary data were available. Although the role of family members of patients with disordered eating behaviours has been discussed in clinical settings, there is no consistent evidence that women with eating disorders were more likely to have sisters than brothers, or to have more females than males in their family (Dolan et al. 1989; Britto et al. 1997). Therefore no adjustment was made in the present study for the number of women in a household. The 1731 women were classified into 5-year age groups $(15-19,20-24,25-29,30-34$ and 35-39 years of age) and then categorised by their current body size as determined by BMI percentiles (5th, 25th, 75th, 95th percentile) calculated for each age group (Table 1). Body-size categories are as follows: lean (BMI $<5$ th percentile); underweight (5th $\leq$ BMI $<25$ th percentile); normal $(25$ th $\leq$ BMI $<75$ th percentile); overweight $(75$ th $\leq$ BMI $<95$ th percentile); obese (BMI $\geq 95$ th percentile).

\section{Outcome measures}

Body-size perception was determined by the woman's response to the question: 'Which best describes your current body size: lean, underweight, normal, overweight, or obese?' We defined 'overestimation' of body size as being a woman's perception of being 'overweight' or 'obese' when her actual current body size was lean, underweight or normal. Therefore, the analyses were only focused on nonobese and non-overweight subjects. We then compared the 'overestimators' with the 'non-overestimators' for the measured and desired BMI, as well as the difference between measured and desired weight, by age and residential area.

Each woman also gave a response regarding her desired weight. To evaluate the desired body size of these women, we calculated the 'desired BMI' of each woman by applying her desired weight and her measured height. The desired BMI was categorised according to the BMI percentiles of her current age group. 'Desire for thinness' was defined as a woman having a desired BMI in the 'lean' or 'underweight' range.

Residential areas were defined by the population size of the woman's current residence. We considered a 'town' $(<50000$ residents) as the base reference area, and compared it with 'metropolitan areas' ( $\geq 1000000$ residents), 'large cities' (150000-1000000 residents) and 'small cities' (50000150000 residents).

\section{Statistical analysis}

All numerical variables including age, height, weight and BMI values were expressed as mean values and SD. One-way ANOVA were used to compare numerical data across different age categories. To compare categorical data, $\chi^{2}$ tests were applied. Logistic regression analyses with multivariate models were used to estimate the adjusted odds ratio OR of 'overestimation' of body size and 'desire for thinness'. $P<0.05$ was considered to be statistically significant. 
All data were analysed with the SAS statistical package (version 8; SAS Institute Inc., Cary, NC, USA).

\section{Results}

The characteristics of the study participants by age group are presented in Table 1. When we compared the measured weight with the desired weight for each age group, the difference between these two variables was smallest in women aged 2529 years. Therefore, we considered this group as a reference for the following analyses. In regard to height, women aged $35-39$ years showed a significantly lower value $(P<0.05)$ compared with the reference group. In regard to mean desired weight, it was significantly lower $(P<0 \cdot 01)$ in the youngest group $(15-19$ years), but was significantly higher $(P<0.05)$ in women aged $35-39$ years. Mean desired BMI was significantly lower $(P<0 \cdot 01)$ in women aged $15-19$ years, but it was significantly higher in women aged $30-34$ years $(P<0 \cdot 05)$, as well as in women aged $35-39$ years $(P<0 \cdot 01)$. In regard to the difference between current and desired weight, there were no significant differences across age groups.

Table 1 also shows the characteristics of the study participants by residential areas. The mean age of the women was similar in the four residential areas. When compared with women residing in towns, BMI values were significantly lower in women from metropolitan areas and large cities $(P<0 \cdot 05)$. The mean desired BMI was significantly lower $(P<0.05)$ in women from metropolitan areas than in women residing in towns. The mean difference between measured and desired weight was smallest in women from large cities, but this was not statistically significant.

Table 2 shows the distributions of perceived body size and desired body size according to actual body size by age groups: $48.4 \%$ of all women perceived themselves as being 'overweight' or 'obese'. In women whose actual body sizes were neither overweight nor obese, the proportion of women who believed that they were 'overweight' or 'obese' (overestimation) was lowest (23.4\%) in the 25-29-year age group: $38.6 \%$ of nonoverweight, non-obese women in the 15-19-year age group overestimated their body sizes. The prevalence of overestimation was $32.7,31.9$ and $36.7 \%$ in non-overweight, non-obese women in the 20-24-year, 30-34-year and 35-39-year age groups, respectively. In all age groups, underweight women were more likely to perceive themselves as being 'normal' than women in the normal category. The proportion of women who had the desire to be in the 'lean' or the 'underweight' category was $53.5,46.9,36.7,36.8$ and $43.1 \%$ in the five age groups. Most women who were currently lean or underweight desired their body size to be 'lean' or 'underweight'.

Table 3 shows the distribution of perceived body size and desired body size according to current body-size categories grouped by the residence area. The proportion of obese women was $4.0 \%$ in metropolitan areas, $3.4 \%$ in large cities, $5.9 \%$ in small cities and $5.9 \%$ in towns. In women who were neither overweight nor obese, the proportion of overestimation was $33.6 \%$ in metropolitan areas, $31.3 \%$ in large cities, $33.9 \%$ in small cities and $33.3 \%$ in towns. The proportion of women whose desired body size was in the 'lean' or the 'underweight' category was $49.8 \%$ in metropolitan areas, $47.4 \%$ in large cities, $41.7 \%$ in small cities and $36.5 \%$ in towns. 
Table 2. Distributions of perceived and desired body size according to actual body size by age groups*

\begin{tabular}{|c|c|c|c|c|c|c|c|c|c|c|c|c|c|c|c|c|c|c|c|c|c|}
\hline \multirow[b]{3}{*}{ Current body size } & \multirow[b]{3}{*}{$n$} & \multicolumn{10}{|c|}{ Perceived body size } & \multicolumn{10}{|c|}{ Desired body size } \\
\hline & & \multicolumn{2}{|c|}{ Lean } & \multicolumn{2}{|c|}{$\begin{array}{l}\text { Under- } \\
\text { weight }\end{array}$} & \multicolumn{2}{|c|}{ Normal } & \multicolumn{2}{|c|}{ Overweight } & \multicolumn{2}{|c|}{ Obese } & \multicolumn{2}{|c|}{ Lean } & \multicolumn{2}{|c|}{ Underweight } & \multicolumn{2}{|c|}{ Normal } & \multicolumn{2}{|c|}{ Overweight } & \multicolumn{2}{|c|}{ Obese } \\
\hline & & $n$ & $\%$ & $n$ & $\%$ & $n$ & $\%$ & $n$ & $\%$ & $n$ & $\%$ & $n$ & $\%$ & $n$ & $\%$ & $n$ & $\%$ & $n$ & $\%$ & $n$ & $\%$ \\
\hline \multicolumn{22}{|l|}{$15-19$ years } \\
\hline Lean & 16 & 5 & $31 \cdot 3$ & 3 & $18 \cdot 8$ & 7 & 43.8 & 1 & $6 \cdot 3$ & - & - & 10 & 62.5 & 5 & $31 \cdot 3$ & 1 & $6 \cdot 3$ & - & - & - & - \\
\hline Underweight & 66 & 5 & $7 \cdot 6$ & 15 & $22 \cdot 7$ & 34 & 51.5 & 10 & $15 \cdot 2$ & 2 & 3.0 & 9 & $13 \cdot 6$ & 49 & $74 \cdot 2$ & 5 & $7 \cdot 6$ & 3 & 4.5 & - & - \\
\hline Normal & 164 & 1 & 0.6 & 7 & 4.3 & 74 & $45 \cdot 1$ & 61 & $37 \cdot 2$ & 21 & $12 \cdot 8$ & 9 & 5.5 & 75 & $45 \cdot 7$ & 80 & $48 \cdot 8$ & - & - & - & - \\
\hline Overweight & 66 & - & - & - & - & 10 & $15 \cdot 2$ & 21 & 31.8 & 35 & $53 \cdot 0$ & 2 & 3.0 & 12 & $18 \cdot 2$ & 48 & $72 \cdot 7$ & 4 & $6 \cdot 1$ & - & - \\
\hline Obese & 15 & - & - & - & - & 1 & $6 \cdot 7$ & 3 & 20.0 & 11 & $73 \cdot 3$ & 1 & $6 \cdot 7$ & 3 & $20 \cdot 0$ & 7 & $46 \cdot 7$ & 4 & $26 \cdot 7$ & - & - \\
\hline \multirow{2}{*}{\multicolumn{22}{|c|}{$20-24$ years }} \\
\hline & & & & & & & & & & & & & & & & & & & & & \\
\hline Lean & 13 & 9 & $69 \cdot 2$ & 3 & $23 \cdot 1$ & 1 & $7 \cdot 7$ & - & - & - & - & 3 & $23 \cdot 1$ & 7 & $53 \cdot 8$ & 3 & $23 \cdot 1$ & - & - & - & - \\
\hline Underweight & 57 & 5 & 8.8 & 12 & $21 \cdot 1$ & 34 & 59.6 & 6 & 10.5 & - & - & 7 & $12 \cdot 3$ & 42 & 73.7 & 8 & 14.0 & - & - & - & - \\
\hline Normal & 138 & - & - & 2 & 1.4 & 74 & $53 \cdot 6$ & 50 & $36 \cdot 2$ & 12 & 8.7 & 1 & 0.7 & 63 & $45 \cdot 7$ & 74 & 53.6 & - & - & - & - \\
\hline Overweight & 56 & - & - & - & - & 3 & 5.4 & 23 & 41.1 & 30 & 53.6 & - & - & 6 & $10 \cdot 7$ & 45 & 80.4 & 5 & 8.9 & - & - \\
\hline Obese & 13 & - & - & - & - & 1 & $7 \cdot 7$ & 1 & 7.7 & 11 & 84.6 & - & - & 1 & $7 \cdot 7$ & 9 & $69 \cdot 2$ & 2 & $15 \cdot 4$ & 1 & 7.7 \\
\hline Total & 277 & 14 & $5 \cdot 1$ & 17 & $6 \cdot 1$ & 114 & $40 \cdot 8$ & 80 & 28.9 & 53 & $19 \cdot 1$ & 11 & 4.0 & 119 & $43 \cdot 0$ & 139 & 50.2 & 7 & 2.5 & 1 & 0.4 \\
\hline \multicolumn{22}{|l|}{$25-29$ years } \\
\hline Lean & 16 & 7 & $43 \cdot 8$ & 6 & 37.5 & 3 & $18 \cdot 8$ & - & - & - & - & 3 & $18 \cdot 8$ & 10 & 62.5 & 3 & $18 \cdot 8$ & - & - & - & - \\
\hline Underweight & 66 & 4 & $6 \cdot 1$ & 18 & $27 \cdot 3$ & 42 & $63 \cdot 6$ & 2 & $3 \cdot 0$ & - & - & 4 & $6 \cdot 1$ & 44 & $66 \cdot 7$ & 18 & $27 \cdot 3$ & - & - & - & - \\
\hline Normal & 166 & 3 & 1.8 & 7 & $4 \cdot 2$ & 100 & $60 \cdot 2$ & 44 & 26.5 & 12 & $7 \cdot 2$ & 2 & $1 \cdot 2$ & 53 & 31.9 & 111 & $66 \cdot 9$ & - & - & - & - \\
\hline Overweight & 66 & - & - & - & - & 2 & 3.0 & 28 & 42.4 & 36 & 54.5 & 1 & 1.5 & 4 & $6 \cdot 1$ & 56 & 84.8 & 5 & $7 \cdot 6$ & - & - \\
\hline Obese & 16 & - & - & - & - & - & - & - & - & 16 & $100 \cdot 0$ & - & - & - & - & 8 & $50 \cdot 0$ & 6 & 37.5 & 2 & 12.5 \\
\hline Total & 330 & 14 & 4.2 & 31 & 9.4 & 147 & 44.5 & 74 & $22 \cdot 4$ & 64 & $19 \cdot 4$ & 10 & 3.0 & 111 & $33 \cdot 6$ & 196 & $59 \cdot 4$ & 11 & 3.3 & 2 & 0.6 \\
\hline \multicolumn{22}{|l|}{$30-34$ years } \\
\hline Lean & 18 & 10 & $55 \cdot 6$ & 7 & 38.9 & 1 & $5 \cdot 6$ & - & - & - & - & 4 & $22 \cdot 2$ & 10 & $55 \cdot 6$ & 4 & $22 \cdot 2$ & - & - & - & - \\
\hline Underweight & 75 & 10 & $13 \cdot 3$ & 20 & $26 \cdot 7$ & 42 & $56 \cdot 0$ & 3 & 4.0 & - & - & 7 & $9 \cdot 3$ & 44 & $58 \cdot 7$ & 24 & $32 \cdot 0$ & - & - & - & - \\
\hline Normal & 186 & 1 & 0.5 & 10 & 5.4 & 89 & $47 \cdot 8$ & 73 & 39.2 & 13 & $7 \cdot 0$ & 4 & $2 \cdot 2$ & 61 & $32 \cdot 8$ & 121 & $65 \cdot 1$ & - & - & - & - \\
\hline Overweight & 75 & - & - & - & - & 3 & 4.0 & 20 & $26 \cdot 7$ & 52 & 69.3 & 1 & 1.3 & 5 & $6 \cdot 7$ & 65 & $86 \cdot 7$ & 4 & 5.3 & - & - \\
\hline Obese & 18 & - & - & - & - & - & - & 1 & $5 \cdot 6$ & 17 & 94.4 & - & - & 1 & 5.6 & 15 & $83 \cdot 3$ & 2 & $11 \cdot 1$ & - & - \\
\hline Total & 372 & 21 & 5.6 & 37 & 9.9 & 135 & $36 \cdot 3$ & 97 & $26 \cdot 1$ & 82 & $22 \cdot 0$ & 16 & $4 \cdot 3$ & 121 & 32.5 & 229 & 61.6 & 6 & 1.6 & - & - \\
\hline \multicolumn{22}{|l|}{$35-39$ years } \\
\hline Lean & 21 & 7 & $33 \cdot 3$ & 8 & 38.1 & 6 & 28.6 & - & - & - & - & 7 & 33.3 & 11 & $52 \cdot 4$ & 3 & $14 \cdot 3$ & - & - & - & - \\
\hline Underweight & 85 & 6 & $7 \cdot 1$ & 28 & 32.9 & 47 & $55 \cdot 3$ & 4 & 4.7 & - & - & 7 & $8 \cdot 2$ & 62 & 72.9 & 16 & $18 \cdot 8$ & - & - & - & - \\
\hline Normal & 213 & 1 & 0.5 & 4 & 1.9 & 95 & 44.6 & 91 & $42 \cdot 7$ & 22 & $10 \cdot 3$ & 6 & $2 \cdot 8$ & 82 & 38.5 & 123 & 57.7 & 2 & 0.9 & - & - \\
\hline Overweight & 85 & - & - & - & - & 1 & $1 \cdot 2$ & 33 & 38.8 & 51 & 60.0 & 1 & 1.2 & 6 & $7 \cdot 1$ & 72 & 84.7 & 6 & $7 \cdot 1$ & - & - \\
\hline Obese & 21 & - & - & - & - & - & - & - & - & 21 & $100 \cdot 0$ & - & - & 1 & 4.8 & 14 & $66 \cdot 7$ & 6 & 28.6 & - & - \\
\hline Total & 425 & 14 & 3.3 & 40 & $9 \cdot 4$ & 149 & $35 \cdot 1$ & 128 & 30.1 & 94 & $22 \cdot 1$ & 21 & 4.9 & 162 & $38 \cdot 1$ & 228 & 53.6 & 14 & 3.3 & - & - \\
\hline
\end{tabular}


Table 3. Distributions of perceived and desired body size according to actual body size by residential area*

\begin{tabular}{|c|c|c|c|c|c|c|c|c|c|c|c|c|c|c|c|c|c|c|c|c|c|c|}
\hline \multirow[b]{3}{*}{ Residential area } & \multirow[b]{3}{*}{ Current body size } & \multicolumn{11}{|c|}{ Perceived body size } & \multicolumn{10}{|c|}{ Desired body size } \\
\hline & & \multicolumn{3}{|c|}{ Lean } & \multicolumn{2}{|c|}{$\begin{array}{l}\text { Under- } \\
\text { weight }\end{array}$} & \multicolumn{2}{|c|}{ Normal } & \multicolumn{2}{|c|}{ Overweight } & \multicolumn{2}{|c|}{ Obese } & \multicolumn{2}{|c|}{ Lean } & \multicolumn{2}{|c|}{$\begin{array}{l}\text { Under- } \\
\text { weight }\end{array}$} & \multicolumn{2}{|c|}{ Normal } & \multicolumn{2}{|c|}{$\begin{array}{l}\text { Over- } \\
\text { weight }\end{array}$} & \multicolumn{2}{|c|}{ Obese } \\
\hline & & $n$ & $n$ & $\%$ & $n$ & $\%$ & $n$ & $\%$ & $n$ & $\%$ & $n$ & $\%$ & $n$ & $\%$ & $n$ & $\%$ & $n$ & $\%$ & $n$ & $\%$ & $n$ & $\%$ \\
\hline \multirow[t]{6}{*}{ Metropolitan } & Lean & 13 & 4 & $30 \cdot 8$ & 5 & 38.5 & 4 & 30.8 & - & - & - & - & 7 & 53.8 & 5 & 38.5 & 1 & $7 \cdot 7$ & - & - & - & - \\
\hline & Underweight & 58 & 3 & $5 \cdot 2$ & 12 & $20 \cdot 7$ & 37 & 63.8 & 4 & 6.9 & 2 & 3.4 & 7 & $12 \cdot 1$ & 43 & 74.1 & 8 & $13 \cdot 8$ & - & - & - & - \\
\hline & Normal & 146 & 1 & 0.7 & 5 & 3.4 & 73 & 50.0 & 55 & 37.7 & 12 & 8.2 & 4 & $2 \cdot 7$ & 64 & 43.8 & 78 & 53.4 & - & - & - & - \\
\hline & Overweight & 49 & - & - & - & - & 6 & $12 \cdot 0$ & 15 & 30.6 & 28 & 57.1 & 1 & 2.0 & 7 & 14.3 & 38 & $77 \cdot 6$ & 3 & $6 \cdot 1$ & - & - \\
\hline & Obese & 11 & - & - & - & - & - & - & 1 & $9 \cdot 1$ & 10 & $90 \cdot 9$ & - & - & - & - & 7 & 63.6 & 4 & $36 \cdot 4$ & - & - \\
\hline & Total & 277 & 8 & 2.9 & 22 & 7.9 & 120 & 43.3 & 75 & $27 \cdot 1$ & 52 & 18.8 & 19 & 6.9 & 119 & 43.0 & 132 & $47 \cdot 7$ & 7 & 2.5 & - & - \\
\hline \multirow[t]{6}{*}{ Large cities } & Lean & 33 & 14 & 42.4 & 10 & $30 \cdot 3$ & 8 & $24 \cdot 2$ & 1 & 3.0 & - & - & 9 & $27 \cdot 3$ & 16 & 48.5 & 8 & $24 \cdot 2$ & - & - & - & - \\
\hline & Underweight & 126 & 12 & 9.5 & 30 & 23.8 & 77 & $61 \cdot 1$ & 7 & 5.6 & - & - & 11 & $8 \cdot 7$ & 87 & 69.0 & 26 & $20 \cdot 6$ & 2 & 1.6 & - & - \\
\hline & Normal & 260 & 1 & 0.4 & 12 & $4 \cdot 6$ & 124 & $47 \cdot 7$ & 98 & 37.7 & 25 & $9 \cdot 6$ & 6 & $2 \cdot 3$ & 109 & 41.9 & 145 & 55.8 & - & - & - & - \\
\hline & Overweight & 95 & - & - & - & - & 2 & $2 \cdot 1$ & 34 & $35 \cdot 8$ & 59 & 62.1 & 3 & $3 \cdot 2$ & 9 & 9.5 & 71 & 74.7 & 12 & $12 \cdot 6$ & - & - \\
\hline & Obese & 18 & - & - & - & - & - & - & 1 & $5 \cdot 6$ & 17 & 94.4 & - & - & 2 & $11 \cdot 1$ & 9 & $50 \cdot 0$ & 6 & 33.3 & 1 & $5 \cdot 6$ \\
\hline & Total & 532 & 27 & $5 \cdot 1$ & 52 & $9 \cdot 8$ & 211 & 39.7 & 141 & 26.5 & 101 & 19.0 & 29 & 5.5 & 223 & 41.9 & 259 & $48 \cdot 7$ & 20 & 3.8 & 1 & 0.2 \\
\hline \multirow[t]{6}{*}{ Small cities } & Lean & 16 & 7 & $43 \cdot 8$ & 6 & 37.5 & 3 & 18.8 & - & - & - & - & 3 & $18 \cdot 8$ & 9 & $56 \cdot 3$ & 4 & $25 \cdot 0$ & - & - & - & - \\
\hline & Underweight & 74 & 7 & 9.5 & 26 & 35.1 & 34 & $45 \cdot 9$ & 7 & 9.5 & - & - & 5 & $6 \cdot 8$ & 53 & 71.6 & 16 & 21.6 & - & - & - & - \\
\hline & Normal & 190 & 3 & 1.6 & 9 & 4.7 & 90 & 47.4 & 72 & 37.9 & 16 & 8.4 & 7 & 3.7 & 77 & 40.5 & 105 & 55.3 & 1 & 0.5 & - & - \\
\hline & Overweight & 90 & - & - & - & - & 6 & 6.7 & 37 & 41.1 & 47 & 52.2 & 1 & $1 \cdot 1$ & 7 & $7 \cdot 8$ & 77 & 85.6 & 5 & $5 \cdot 6$ & - & - \\
\hline & Obese & 23 & - & - & - & - & - & - & 3 & $13 \cdot 0$ & 20 & 87.0 & 1 & 4.3 & 1 & $4 \cdot 3$ & 19 & 82.6 & 1 & 4.3 & 1 & $4 \cdot 3$ \\
\hline & Total & 393 & 17 & $4 \cdot 3$ & 41 & $10 \cdot 4$ & 133 & 33.8 & 119 & $30 \cdot 3$ & 83 & $21 \cdot 1$ & 17 & 4.3 & 147 & 37.4 & 221 & $56 \cdot 2$ & 7 & 1.8 & 1 & 0.3 \\
\hline \multirow[t]{6}{*}{ Towns } & Lean & 22 & 13 & 59.1 & 6 & $27 \cdot 3$ & 3 & 13.6 & - & - & - & - & 5 & $27 \cdot 7$ & 15 & 68.2 & 2 & $9 \cdot 1$ & - & - & - & - \\
\hline & Underweight & 91 & 8 & 8.8 & 25 & 27.5 & 51 & 56.0 & 7 & $7 \cdot 7$ & - & - & 11 & $12 \cdot 1$ & 54 & 59.3 & 26 & 28.6 & - & - & - & - \\
\hline & Normal & 271 & 1 & 0.4 & 4 & 1.5 & 145 & 53.5 & 94 & 34.7 & 27 & $10 \cdot 0$ & 7 & $2 \cdot 6$ & 88 & 32.5 & 175 & $64 \cdot 6$ & 1 & 0.4 & - & - \\
\hline & Overweight & 114 & - & - & - & - & 5 & 4.4 & 39 & $34 \cdot 2$ & 70 & 61.4 & 1 & 0.9 & 9 & 7.9 & 101 & $86 \cdot 6$ & 3 & $2 \cdot 6$ & - & - \\
\hline & Obese & 31 & - & - & - & - & 2 & 6.5 & - & - & 29 & 93.5 & - & - & 3 & $9 \cdot 7$ & 19 & $61 \cdot 3$ & 7 & $22 \cdot 6$ & 2 & 6.5 \\
\hline & Total & 529 & 22 & $4 \cdot 2$ & 35 & $6 \cdot 6$ & 206 & 38.9 & 140 & 26.5 & 126 & 23.8 & 24 & 4.5 & 169 & 31.9 & 323 & $61 \cdot 1$ & 11 & $2 \cdot 1$ & 2 & 0.4 \\
\hline
\end{tabular}

For details of definition of terms, p. 1155. 
Table 4 shows the measured and desired BMI, as well as the difference between measured and desired weight by the degree of 'overestimation'. In regard to age, the proportion of 'overestimators' in women aged 25-29 years was the smallest $(23.4 \%)$ among all age groups, compared with the 15-19-year group where $38.6 \%$ were 'overestimators'. In regard to residential areas, there were fewer 'overestimators' in large cities $(31.3 \%)$ than in small cities $(33.3 \%)$. In total, $32.8 \%$ of women were considered as 'overestimators'. In regard to the difference between the measured and desired BMI, the measured BMI of 'overestimators' was significantly higher than that of 'non-overestimators' $(P<0 \cdot 01)$ regardless of age or residential areas. The mean desired BMI was also higher in the 'overestimators', but the difference between the two groups was relatively small compared with the differences in the measured BMI between the two groups across all age groups and residential areas. The desired BMI was similar in 'overestimators' and 'non-overestimators' in women in the 15-19-year age group, and their desired BMI was below $18.5 \mathrm{~kg} / \mathrm{m}^{2}$ in both 'overestimators' and 'non-overestimators'. Differences between measured and desired weight in 'overestimators' were similar across all age groups, and were significantly $(P<0 \cdot 01)$ larger compared with 'non-overestimators'. Significant differences $(P<0.01)$ in the gap between measured and desired weight between 'overestimators' and 'non-overestimators' were seen in all residential areas.

We calculated the OR for the related variables to 'overestimation' in non-obese, non-overweight women using a multivariate logistic regression model adjusted for the current BMI (Table 5). In this analysis, 427 women were defined as 'overestimators'. The OR for 'overestimation' were 2.79 (95\% CI $1.76,4.43 ; P<0.01)$ in the $15-19$-year age group and $0.75(95 \%$ CI $0.47,1.18 ; P=0.21)$ in the 35-39-year age group, compared with the 25-29-year age group. The OR for 'overestimation' increased with the population size of the residence area, but was not significant.

We calculated the OR for the related variables for 'desire for thinness' with all subjects, using a multivariate logistic regression model adjusted for the current BMI (Table 6). In regard to age group, 756 were defined as having 'desire for thinness' in the analyses. The OR for 'desire for thinness'

Table 4. Comparisons of the measured and desired body mass index, and 'difference between measured and desired weight' between 'overestimators' and 'non-overestimators' by age group and residential area†

\begin{tabular}{|c|c|c|c|c|c|c|c|}
\hline \multirow[b]{2}{*}{ Variable } & \multirow[b]{2}{*}{$n$} & \multicolumn{2}{|c|}{$\begin{array}{l}\text { Measured BMI } \\
\left(\mathrm{kg} / \mathrm{m}^{2}\right)\end{array}$} & \multicolumn{2}{|c|}{$\begin{array}{l}\text { Desired BMI } \\
\left(\mathrm{kg} / \mathrm{m}^{2}\right)\end{array}$} & \multicolumn{2}{|c|}{$\begin{array}{c}\text { Difference } \\
\text { between } \\
\text { measured and } \\
\text { desired weight } \\
(\mathrm{kg})\end{array}$} \\
\hline & & Mean & SD & Mean & SD & Mean & SD \\
\hline \multicolumn{8}{|l|}{ Age group } \\
\hline \multicolumn{8}{|l|}{$15-19$ years } \\
\hline Overestimators & 95 & $20 \cdot 0^{\star *}$ & 0.9 & $18 \cdot 4$ & $1 \cdot 1$ & $4 \cdot 0^{\star \star}$ & $2 \cdot 3$ \\
\hline Non-overestimators & 151 & $18 \cdot 9$ & $1 \cdot 3$ & $18 \cdot 3$ & 1.3 & 1.4 & 3.4 \\
\hline \multicolumn{8}{|l|}{$20-24$ years } \\
\hline Overestimators & 68 & $20 \cdot 3^{\star *}$ & $1 \cdot 0$ & $18 \cdot 7^{*}$ & $1 \cdot 1$ & $4 \cdot 2^{\star \star}$ & $2 \cdot 5$ \\
\hline Non-overestimators & 140 & 18.9 & 1.5 & $18 \cdot 5$ & 1.0 & $1 \cdot 1$ & 3.5 \\
\hline \multicolumn{8}{|l|}{$25-29$ years } \\
\hline Overestimators & 58 & $20 \cdot 6^{\star *}$ & 0.9 & $19 \cdot 0^{*}$ & 1.0 & $4 \cdot 0^{\star *}$ & $2 \cdot 2$ \\
\hline Non-overestimators & 190 & $18 \cdot 9$ & $1 \cdot 3$ & $18 \cdot 7$ & 0.9 & 0.4 & $2 \cdot 7$ \\
\hline \multicolumn{8}{|l|}{$30-34$ years } \\
\hline Overestimators & 89 & $20 \cdot 1^{\star *}$ & $1 \cdot 0$ & $19 \cdot 4^{\star *}$ & 1.0 & $4 \cdot 2^{\star \star}$ & $2 \cdot 4$ \\
\hline Non-overestimators & 190 & $19 \cdot 5$ & 1.4 & $19 \cdot 1$ & $1 \cdot 1$ & 0.0 & 3.5 \\
\hline \multicolumn{8}{|l|}{$35-39$ years } \\
\hline Overestimators & 117 & $20 \cdot 6^{\star *}$ & $1 \cdot 1$ & $19 \cdot 8^{\star \star}$ & $1 \cdot 0$ & $4 \cdot 3^{\star \star}$ & $2 \cdot 2$ \\
\hline Non-overestimators & 202 & 19.5 & 1.4 & $19 \cdot 3$ & $1 \cdot 2$ & 0.5 & $3 \cdot 1$ \\
\hline \multicolumn{8}{|l|}{ Residential area } \\
\hline \multicolumn{8}{|l|}{ Metropolitan } \\
\hline Overestimators & 73 & $20 \cdot 6^{\star \star}$ & 1.1 & $19 \cdot 1^{* *}$ & 1.0 & $3 \cdot 8^{\star *}$ & $2 \cdot 0$ \\
\hline Non-overestimators & 144 & $19 \cdot 1$ & 1.4 & $18 \cdot 6$ & 1.0 & $1 \cdot 3$ & 2.9 \\
\hline \multicolumn{8}{|l|}{ Large cities } \\
\hline Overestimators & 131 & $20 \cdot 9^{\star \star}$ & $1 \cdot 3$ & $19 \cdot 0^{*}$ & $1 \cdot 1$ & $4 \cdot 5^{\star *}$ & $2 \cdot 5$ \\
\hline Non-overestimators & 288 & $18 \cdot 8$ & $1 \cdot 3$ & $18 \cdot 8$ & $1 \cdot 1$ & 0.1 & 3.4 \\
\hline \multicolumn{8}{|l|}{ Small cities } \\
\hline Overestimators & 95 & $20 \cdot 8^{\star \star}$ & $1 \cdot 2$ & $19 \cdot 0$ & 1.3 & $4 \cdot 4^{\star \star}$ & $2 \cdot 6$ \\
\hline Non-overestimators & 185 & $19 \cdot 1$ & 1.4 & $18 \cdot 9$ & $1 \cdot 2$ & 0.6 & 3.4 \\
\hline \multicolumn{8}{|l|}{ Towns } \\
\hline Overestimators & 128 & $20 \cdot 9^{\star \star}$ & 1.1 & $19 \cdot 3^{*}$ & $1 \cdot 2$ & $3.9^{\star \star}$ & $2 \cdot 0$ \\
\hline Non-overestimators & 256 & $19 \cdot 3$ & 1.4 & $19 \cdot 0$ & 1.3 & 0.8 & $3 \cdot 1$ \\
\hline \multicolumn{8}{|l|}{ Total } \\
\hline Overestimators & 427 & $20 \cdot 8^{\star *}$ & $1 \cdot 2$ & $19 \cdot 1^{\star *}$ & $1 \cdot 2$ & $4 \cdot 2^{\star *}$ & $2 \cdot 3$ \\
\hline Non-overestimators & 873 & $19 \cdot 1$ & 1.4 & $18 \cdot 8$ & $1 \cdot 2$ & 0.6 & $3 \cdot 3$ \\
\hline
\end{tabular}

Mean value was significantly different from that of 'non-overestimators' in the same category: * $P<0.05,{ }^{\star *} P<0.01$.

†For details of definition of terms, see p. 1155. 
Table 5. Multivariate logistic regression analyses for 'overestimation' by age group and residential area*

(OR and $95 \% \mathrm{Cl}$ )

\begin{tabular}{lllr}
\hline Variable & OR & \multicolumn{1}{c}{$95 \% \mathrm{Cl}$} & \multicolumn{1}{c}{$P$} \\
\hline Age group & & & \\
15-19 years & 2.79 & $1.76,4.43$ & $<0.01$ \\
$20-24$ years & 1.57 & $0.96,2.56$ & 0.07 \\
$25-29$ years & 1.00 & Referent & \\
30-34 years & 0.98 & $0.62,1.57$ & 0.94 \\
25-39 years & 0.75 & $0.47,1.19$ & 0.21 \\
Residential area & & & \\
Metropolitan & 1.41 & $0.91,2.17$ & 0.12 \\
Large cities & 1.42 & $0.98,2.05$ & 0.06 \\
Small cities & 1.25 & $0.84,1.86$ & 0.28 \\
Towns & 1.00 & Referent & \\
\hline
\end{tabular}

* Analyses were adjusted for the current BMI; subjects were restricted to non-obese and non-overweight women in the analyses. For details of definition of terms, see p. 1155.

Table 6. Multivariate logistic regression analyses for 'desire for thinness' by age group, residential area and overestimation of body size*

(OR and $95 \% \mathrm{Cl}$ )

\begin{tabular}{lccc}
\hline Variable & OR & $95 \% \mathrm{Cl}$ & $P$ \\
\hline Age group & & & \\
$15-19$ years & 2.26 & $1.57,3.24$ & $<0.01$ \\
$20-24$ years & 2.05 & $1.41,2.98$ & $<0.01$ \\
$25-29$ years & 1.00 & Referent & \\
$30-34$ years & 1.31 & $0.92,1.86$ & 0.13 \\
$25-39$ years & 2.74 & $1.93,3.89$ & $<0.01$ \\
Residential area & & & \\
$\quad$ Metropolitan & 1.47 & $1.05,2.07$ & 0.03 \\
$\quad$ Large cities & 1.31 & $0.98,1.74$ & 0.07 \\
Small cities & 1.19 & $0.88,1.63$ & 0.26 \\
$\quad$ Towns & 1.00 & Referent & \\
Overestimation of body size† & & \\
$\quad$ Yes & 1.33 & $1.03,1.70$ & 0.03 \\
$\quad$ No & 1.00 & Referent & \\
\end{tabular}

* Analyses were adjusted for the current BMI. For details of definition of terms, see p. 1155.

†Subjects were restricted to non-obese and non-overweight women in the analyses.

were 2.26 (95\% CI $1.57,3 \cdot 24 ; P<0.01)$ in the $15-19$-year age group and $2.74(95 \%$ CI $1.93,3.89 ; P<0.01)$ in the 35-39year age group, compared with the 25-29-year age group. In regard to residential area, 747 women were defined as having 'desire for thinness'. The OR for 'desire for thinness' increased as the population size increased. The OR was significantly higher in women who resided in metropolitan areas (OR 1.47 (95\% CI 1.05, 2.07); $P=0.03$ ). In addition, women who had overestimated their body size $(n 427)$ were 1.33 times more likely to have 'desire for thinness' than those who did not (OR 1.33 (95\% CI 1.03, 1.70); $P=0.03$ ).

\section{Discussion}

The present results clearly demonstrated that (1) both measured and desired BMI of women living in metropolitan areas were significantly lower compared with towns, (2) body-size perception was the most accurate in women aged
25-29 years, (3) significant 'overestimation' of body size was observed in women aged 15-19 years, (4) both young and middle-aged women had significantly higher 'desire for thinness', (5) women living in metropolitan areas had significantly higher 'desire for thinness', and (6) women who overestimated their body sizes were more likely to desire thinner body sizes than those who did not. However, findings from our present study did not support one of our hypotheses that women living in larger cities were more likely to overestimate their body size than women living in smaller towns. Women living in larger cities tended to overestimate their body sizes, but no significant association was observed.

The present study demonstrated that 'desire for thinness' is not only observed in young Japanese women, but also in middle-aged women. Pubertal changes and weight gains in young women have a significant effect on the development of one's body image and body satisfaction (Cash \& Pruzinsky, 2002; Suka et al. 2005). This is also observed in teenage girls and female college students in Japan, who have the strong 'desire for thinness', despite the fact that they are not overweight (Kaneda et al. 2004; Wardle et al. 2005). Our findings on increased 'desire for thinness' in young women are in accordance with these existing studies. However, research on middle-aged Japanese women in relation to weight perceptions and 'desire for thinness' has been limited. In the UK, for example, 'desire for thinness' of elderly women was reported to be equivalent to that of young women (Hetherington \& Burnett, 1994). In addition, Allaz et al. (1998) found that many older women in Switzerland continued to engage in dieting, despite the fact that they were of normal weight, reflecting the continued pressure felt by women to lose weight. In the present study, the measured body weight in women aged 35-39 years was significantly higher compared with the reference group of 25-29 years. Therefore, we assume that weight gain due to ageing brings dissatisfaction in current body size, because thinness is a standard definition of attractiveness for women at all ages.

There are several points that should be considered when interpreting the present results. First, the present study is a cross-sectional observation which makes it difficult to determine the causal relationships even though the results were generated from nationally representative samples. Second, the BMI cut-off points we applied in the present study were neither the international standard nor the Japanese standard developed by the Japan Society for the Study of Obesity (Matsuzawa et al. 2000). These standards were both established based on risks of chronic diseases or mortality. In other words, these are more obesity-oriented. The present study, on the other hand, is aimed at identifying extravagant weight-control behaviours of young Japanese women, in which the increased prevalence of underweight (BMI $<18.5 \mathrm{~kg} / \mathrm{m}^{2}$ ) and extreme underweight (BMI $<17.0 \mathrm{~kg} / \mathrm{m}^{2}$ ) has been the major public health problem (Takimoto et al. 2004). The number of overweight women in the young generation are rather scarce, contrary to the worldwide obesity epidemic. These facts raised the assumption that the BMI distribution curve of this population group was likely to skew to the lower end than the upper. In addition, we had to consider significant change in the BMI distribution curve with advancing age. We assume that the application of neither the international nor the Japanese standard cut-off points 
disables quantitative comparisons with corresponding studies from other population groups. However, the increase in the prevalence of underweight is specific to this population group in the first place. Therefore, the use of relative BMI cut-off points to define body-size categories is an appropriate approach to learn specific characteristics of this particular population group. The findings suggest the nature of excessive weight concerns of young women in Japan. Underweight was also evident in this population group; thus efforts to control such health-risk behaviours of young women at a national level are urgent.

Women are more likely to experience less bodily satisfaction and are more likely to have lower self esteem and negative consequences (i.e. eating disorders) compared with men, regardless of age (Cash \& Pruzinsky, 2002). In the present study, despite the fact that a large proportion of women were not overweight, they overestimated their body size and wanted to become even leaner. According to the 2002 NNSJ report, $12.9 \%$ of underweight (BMI $<18.5 \mathrm{~kg} / \mathrm{m}^{2}$ ) women aged 15 years and older were actually trying to lose weight. Dieting behaviours among underweight women were prominent in women aged 15-19 years $(41.0 \%)$, and the rate decreased with advanced age. Furthermore, $52.0 \%$ of normal-weight (BMI 18.5-24.9 $\mathrm{kg} / \mathrm{m}^{2}$ ) women were trying to lose weight, and the prevalence rate of women aged 15-19 years, 20-29 years and 30-39 years were 68.6, 63.8 and $55.4 \%$, respectively. These results suggest that the increase in the prevalence of underweight among young Japanese women is partly due to unnecessary weight control of non-obese individuals. It has been reported that body image concerns during adolescence are related to lower self esteem and are related to increased risks for eating disorders (Cash \& Pruzinsky, 2002). In addition, weight-concerned mothers have an impact on their child's behaviours, such as negative weight concerns and unhealthy dieting behaviours (Francis \& Birch, 2005). Health professionals need to disseminate information on the risks of unhealthy weight-control behaviours and the benefits of a healthy diet, especially to women of reproductive age. Further studies are needed to identify how overestimation of body sizes and strong 'desire for thinness' observed in this very lean population group are related to poor health of young Japanese women.

\section{Acknowledgements}

The present study is funded by Research on Children and Families (the Ministry of Health, Labour and Welfare) and a research grant for Surveys on Nutrition and Diet of the Japanese People (National Institute of Health and Nutrition).

\section{References}

Allaz AF, Bernstein M, Rouget P, Archinard M \& Morabia A (1998) Body weight preoccupation in middle-aged and ageing women: a general population survey. Int J Eat Disord 23, 287-294.

Blum M, Harris SS, Must A, Phillips SM, Rand WM \& DawsonHughes B (2001) Weight and body mass index at menarche are associated with premenopausal bone mass. Osteoporos Int 12, $588-594$.
Britto DJ, Meyers DH, Smith JJ \& Palmer RL (1997) Anorexia nervosa and bulimia nervosa: sibling sex ratio and birth rank - a catchment area study. Int J Eat Disord 21, 335-340.

Calle EE \& Thun MJ (2004) Obesity and cancer. Oncogene 23, $6365-6378$.

Cash TF \& Pruzinsky T (2002) Body Image: A Handbook of Theory, Research, and Clinical Practice. New York: The Guilford Press.

Chan JM, Rimm EB, Colditz GA, Stampfer MJ \& Willett WC (1994) Obesity, fat distribution, and weight gain as risk factors for clinical diabetes in men. Diabetes Care 17, 961-969.

Dolan BM, Evans C \& Lacey JH (1989) Family composition and social class in bulimia. A catchment area study of a clinical and a comparison group. J Nerv Ment Dis 177, 267-272.

Edwards LE, Alton IR, Barrada MI \& Hakanson EY (1979) Pregnancy in the underweight woman. Course, outcome, and growth patterns of the infant. Am J Obstet Gynecol 135, 297-302.

Francis LA \& Birch LL (2005) Maternal influences on daughters' restrained eating behavior. Health Psychol 24, 548-554.

Hetherington MM \& Burnett L (1994) Ageing and the pursuit of slimness: dietary restraint and weight satisfaction in elderly women. Br J Clin Psychol 33, 391-400.

Kaneda F, Sugano S, Sano A, Nishida M, Yoshiike N \& Yamamoto S (2004) Systematic review of the frequency of "thinness" among children and adolescents in Japan. Jpn J Nutr Diet 62, 347-360.

Kuchler F \& Variyam JN (2003) Mistakes were made: misperception as a barrier to reducing overweight. Int J Obes Relat Metab Disord 27, 856-861.

McLaren L \& Gauvin L (2003) Does the 'average size' of women in the neighborhood influence a woman's likelihood of body dissatisfaction? Health Place 9, 327-335.

Manson JE, Colditz GA, Stampfer MJ, Willett WC, Rosner B, Monson RR, Speizer FE \& Hennekens CH (1990) A prospective study of obesity and risk of coronary heart disease in women. $N$ Engl J Med 322, 882-889.

Matsuzawa Y, Inoue S, Ikeda Y, et al. (2000) New criteria for obesity and obesity disease (article in Japanese). Obes Res (Japan) 6, $18-28$.

Ministry of Health, Labour and Welfare (2000a) Health Japan 21: Report on Health Japan 21 Plan Study Committee \& Health Japan 21 Plan Development Committee. Tokyo: Taiyou Bijyutsu Co.

Ministry of Health, Labour and Welfare (2000b) Annual Report of the National Nutrition Survey in 1998. Tokyo: Daiichi Publishing Co.

Ministry of Health, Labour and Welfare (2004) Annual Report of the National Nutrition Survey in 2002. Tokyo: Daiichi Publishing Co.

Paeratakul S, White MA, Williamson DA, Ryan DH \& Bray GA (2002) Sex, race/ethnicity, socioeconomic status, and BMI in relation to self-perception of overweight. Obes Res 10, 345-350.

Russell JD, Mira M, Allen BJ, Stewart PM, Vizzard J, Arthur B \& Beumont PJ (1994) Protein repletion and treatment in anorexia nervosa. Am J Clin Nutr 59, 98-102.

Suka M, Sugimori H, Yoshida K, Kanayama H, Sekine M, Yamagami T \& Kagamimori S (2005) Body image and body satisfaction play important roles in the path to dieting behavior in Japanese preadolescents: The Toyama Birth Cohort Study. Environ Health Prev Med 10, 324-330.

Takimoto H, Yoshiike N, Kaneda F \& Yoshita K (2004) Thinness among young Japanese women. Am J Public Health 94, 1592-1595.

Talamayan KS, Springer AE, Kelder SH, Gorospe EC \& Joye KA (2006) Prevalence of overweight misperception and weight control behaviors among normal weight adolescents in the United States. Scientific World Journal 26, 365-373.

Tsugane S, Sasaki S \& Tsubono Y (2002) Under- and overweight impact on mortality among middle-aged Japanese men and women: a $10-\mathrm{y}$ follow-up of JPHC study cohort I. Int $J$ Obes Relat Metab Disord 26, 529-537. 
Wardle J, Haase AM \& Steptoe A (2005) Body image and weight control in young adults: international comparisons in university students from 22 countries. Int J Obes (Lond) 30, 644-651.

Watanabe H (2003) Research on Current Status of Anorexia Nervosa and its Policy: The 2002 Report no. H13-child-015Tokyo: Ministry of Health, Labour and Welfare, Maternal and Child Health Division.

Wilson PW, D'Agostino RB, Sullivan L, Parise H \& Kannel WB (2002) Overweight and obesity as determinants of cardiovascular risk: the Framingham experience. Arch Intern Med 162, $1867-1872$.

World Health Organization (2003) Global Strategy on Diet, Physical Activity and Health: Obesity and Overweight. Accessed 19 July 2006. http://www.who.int/hpr/NPH/docs/ gs_obesity.pdf

Yoshiike N, Seino F, Tajima S, Arai Y, Kawano M, Furuhata T \& Inoue $S$ (2002) Twenty-year changes in the prevalence of overweight in Japanese adults: The National Nutrition Survey 1976-95. Obes Rev 3, 183-190. 